\title{
Neuromyelitis Optica: A Diffusional Kurtosis Imaging Study
}

T.M. Doring, F.C.R. Lopes, T.T.A. Kubo, G. Tukamoto, M.C. Kimura, R.M. Strecker, R.C. Domingues, and E.L. Gasparetto O-

\begin{abstract}
BACKGROUND AND PURPOSE: Conventional MR imaging typically yields normal images of the brain or indicates lesions in areas of high aquaporin expression in patients with neuromyelitis optica. Diffusional kurtosis imaging was applied in patients with neuromyelitis optica to determine whether this technique could detect alterations in diffusion and diffusional kurtosis parameters in normal-appearing white matter and to explore the relationship between diffusional kurtosis imaging and DTI parameters.
\end{abstract}

MATERIALS AND METHODS: Thirteen patients with neuromyelitis optica and 13 healthy controls underwent MR imaging of the brain with conventional and diffusional kurtosis imaging sequences. Tract-based spatial statistics and region-of-interest-based analyses were conducted to identify differences between patients with neuromyelitis optica and controls through conventional DTI and diffusional kurtosis imaging parameters. The parameters were correlated to determine the potential relationship between them.

RESULTS: Compared with healthy controls, several diffusional kurtosis imaging and DTI parameters were altered in various fiber tracts of patients with neuromyelitis optica $(P<.05)$. A significant decrease $(P<.05)$ in radial kurtosis was observed in the corpus callosum and anterior corona radiata and left optic radiation. Differences $(P<.1)$ in mean kurtosis were found in patients with neuromyelitis optica. We found a negative correlation between diffusional kurtosis imaging (radial kurtosis, axial kurtosis, mean kurtosis) and the corresponding DTI parameters (radial diffusivity, axial diffusivity, mean diffusivity). Positive correlations were found for radial kurtosis and mean kurtosis with fractional anisotropy.

CONCLUSIONS: This study demonstrated differences in conventional diffusion and diffusional kurtosis parameters, especially radial kurtosis, in the normal-appearing white matter of patients with neuromyelitis optica compared with healthy controls. Larger studies of patients with neuromyelitis optica should be performed to assess the potential clinical impact of these findings.

ABBREVIATIONS: $\mathrm{AD}=$ axial diffusivity; $\mathrm{AK}=$ axial kurtosis; $\mathrm{CC}=$ corpus callosum; $\mathrm{DKI}=$ diffusional kurtosis imaging; $\mathrm{FA}$, fractional anisotropy; $\mathrm{MD}=$ mean diffusivity; $\mathrm{MK}=$ mean kurtosis; $\mathrm{NMO}=$ neuromyelitis optica; $\mathrm{OR}=$ optic radiation; $\mathrm{RD}=$ radial diffusivity; $\mathrm{RK}=$ radial kurtosis; TBSS = tract-based spatial statistics

$\mathbf{N}$ euromyelitis optica (NMO) is a severe, recurrent demyelinating disease that typically affects the optic nerve and spinal cord. ${ }^{1,2}$ In patients with NMO, conventional MR imaging typically yields images with normal findings of the brain or indicates lesions in areas of high aquaporin expression. ${ }^{3}$ Studies by using diffusion-tensor MR imaging, which depicts several diffusion pa-

Received March 13, 2014; accepted after revision June 2.

From the Universidade Federal Rio de Janeiro (T.M.D., F.C.R.L., G.T., E.L.G.), Rio de Janeiro, Brazil; Clínica de Diagnóstico Por Imagem and Multi-imagem (T.M.D.,

F.C.R.L., T.T.A.K., G.T., M.C.K., R.C.D., E.L.G.), Diagnósticos da América, Rio de Janeiro, Brazil; and Siemens Healthcare (R.M.S.), São Paulo, Brazil.

This work was supported by Coordenação de Aperfeiçoamento de Pessoal de Nível Superior (CAPES).

Please address correspondence to Thomas Doring, MS, Avenue Das Américas 4666, Sala 323, CEP 22640102 Rio de Janeiro, Brazil; e-mail: thomas.doring@gmail.com

O- Indicates open access to non-subscribers at www.ajnr.org

三 Indicates article with supplemental on-line table.

http://dx.doi.org/10.3174/ajnr.A4050 rameters, have identified extensive damage in the normal-appearing white matter in patients with $\mathrm{NMO}$, affecting the pyramidal tract, optic radiation (OR), and corpus callosum (CC). ${ }^{4,5}$

Diffusional kurtosis imaging (DKI) is an extension of the DTI technique that allows the simultaneous estimation of diffusion and kurtosis parameters. ${ }^{6}$ The DTI model assumes that diffusion occurs in a random unrestricted way, following a Gaussian diffusion probability distribution function. However, the complexity of tissue is the reason that water diffusion deviates from that strict Gaussian behavior and occurs in a restricted way. DKI considers this in its model by quantifying this deviation, the diffusional kurtosis; thus, it is a more accurate description of restricted diffusion than DTI and may provide additional biomarkers in various disease states. ${ }^{7,8}$ In combined DTI and DKI acquisitions, at least 15 distinct nonlinear diffusion directions (gradients), with at least 3 distinct b-values, ${ }^{9}$ are obtained. Typical b-values for DKI in the brain are 0,1000 , and $2000 \mathrm{~s} / \mathrm{mm}^{2}$. An advantage of DKI is that it apparently accounts for fiber-crossing, whereas DTI fails in 
this respect. ${ }^{10}$ Q-ball imaging, a previously described technique that also accounts for fiber crossing, requires numerous encoding directions and even greater b-values, resulting in high acquisition times. ${ }^{11,12}$ Therefore, DKI might be useful when shorter acquisition times are needed because it requires only 15 independent directions and lower b-values.

Mean kurtosis (MK), which provides a measure of the overall kurtosis, is the main DKI parameter of interest in an isotropic medium. When diffusion becomes anisotropic (eg, within nerve fibers), directional kurtosis metrics, such as axial kurtosis (AK) and radial kurtosis (RK), can be derived. As in conventional DTI, $\mathrm{AK}$ is defined as the kurtosis along the principal eigenvector, whereas RK is the kurtosis perpendicular to the principal eigenvector. Kurtosis parameters have been shown to correlate negatively with DTI parameters. ${ }^{13}$ In a previous study, ${ }^{14}$ the DKI parameters, especially RK, showed high sensitivity to the degree of myelination of the brain in rats. RK was increased due to a more restricted diffusion perpendicular to the axon during myelination. Conversely, it is expected that RK will decrease when diffusion becomes less restricted during demyelination. Lätt et $\mathrm{al}^{15}$ recently estimated and compared the regional DKI values of healthy brain tissues with DKI values obtained from previous studies of different pathologies. In another study in which DKI was applied after stroke onset, ${ }^{16}$ higher MK values were observed in ischemic WM, which eventually progressed to gliosis, compared with ischemic WM that maintained a normal appearance. These findings suggest the usefulness of DKI for predicting the outcome of ischemic tissue. Finally, DKI parameters were found to be more effective than conventional DTI parameters for differentiating high- and low-grade gliomas. ${ }^{17}$

To date, few studies have assessed the clinical application of DKI. The purpose of this study was to evaluate the brains of patients with NMO by DKI, to determine whether DKI can detect differences in DKI parameters compared with healthy controls and to explore the relationship between DKI and conventional DTI parameters.

\section{MATERIALS AND METHODS \\ Subjects}

Thirteen patients diagnosed with NMO (mean age, $35 \pm 14$ years; age range, $14-64$ years; 6 females and 7 males) were selected from the Clinical Demyelinating Disease data base of our university hospital. All patients fulfilled the revised Criteria of 2006 of Wingerchuk et al. ${ }^{1}$ Thirteen demographically matched healthy controls (mean age, $37 \pm$ 13 years; age range, 15-57 years), free of neurologic or psychiatric disorders, were also enrolled. All subjects gave written informed consent, and the study was approved by the institutional review board.

\section{MR Imaging Acquisition}

All subjects underwent MR imaging at 3T (Tim Trio; Siemens, Erlangen, Germany). In addition to standard sagittal T1WI and axial T2WI (FLAIR) sequences, a work-in-progress DKI sequence was acquired with 30 gradient directions and the following parameters: bvalues of $1 b=0 \mathrm{~s} / \mathrm{mm}^{2}, 30 b=1000 \mathrm{~s} / \mathrm{mm}^{2}$, and $30 b=2000 \mathrm{~s} / \mathrm{mm}^{2}$; $\mathrm{TR} / \mathrm{TE}=5300 / 90 \mathrm{~ms} ; \mathrm{FOV}=220 \mathrm{~mm} ; 45$ sections; matrix $=82 \times$ 82 ; section thickness $=2.7 \mathrm{~mm}$; voxel size $=2.7 \mathrm{~mm}^{3}$ isotropic; bipolar gradient scheme; bandwidth $=3048 \mathrm{~Hz} / \mathrm{Px}$; parallel imaging factor $=2$; echo spacing $=0.59 \mathrm{~ms}$; no gap.

\section{DTI and DKI Parametric Map Calculations}

DKI raw datasets were processed off-line on a workstation. To calculate the diffusion and kurtosis parametric maps, a customwritten software in Matlab (MathWorks, Natick, Massachusetts) ${ }^{6}$ was used. The kurtosis model, as described previously, ${ }^{5,6}$ was fitted to the diffusion-weighted signal intensities in each voxel by nonlinear least-squares minimization. The fitted kurtosis tensor was used to calculate $\mathrm{AK}, \mathrm{RK}$, and $\mathrm{MK}$, as described by Tabesh et al. ${ }^{6}$ Fractional anisotropy (FA), mean diffusion (MD), axial diffusion $(A D)$, and radial diffusion $(\mathrm{RD})$ were calculated from the diffusion tensor in the kurtosis model by using conventional equations. ${ }^{6}$ After this, parametric maps were constructed for each parameter (Fig 1).

\section{Data Analysis}

Tract-Based Spatial Statistics for Group Analysis. First, all subject data were analyzed statistically by using tract-based spatial statistics (TBSS), a software tool within the fMRI of the Brain Software Library (FSL; http://www.fmrib.ox.ac.uk/fsl). ${ }^{18}$ All of the FA maps of subjects were aligned by using nonlinear registration. Then, a mean FA image was created and thinned to generate a mean FA skeleton, which represents the center of all tracts common to the group. We registered each subject's parametric diffusion (MD, RD, and $\mathrm{AD}$ ) and kurtosis images (MK, RK, and $\mathrm{AK}$ ) into the same space as the FA, applying the same nonlinear registration used for the previously processed FA images, and projected them onto the skeleton. To perform group comparisons (patients with NMO versus healthy controls), we determined voxelwise cross-subject statistics for each parameter, by using permutation-based inference with 5000 permutations and thresholdfree cluster enhancement with a threshold of $P<.05$, corrected for multiple comparisons, by using family-wise error correction. Areas with significantly altered parameters in patients with NMO compared with healthy controls were identified by the Johns Hopkins University White Matter Tractography Atlas (http:// cmrm.med.jhmi.edu/) (Fig 2).

Region-of-Interest-Based Group Analysis. Second, a region-ofinterest-driven analysis was performed by 2 experienced neuroscientists (F.C.R.L. and T.M.D., each with 7 years of experience) by placing hand-drawn ROIs on the splenium and genu of the CC, corticospinal tract in the cerebral peduncles, and ORs on the B0 maps of each subject by using OsiriX (Version 4.1.2; http:// www.osirix-viewer.com). The purpose of this second a priori analysis was to corroborate the TBSS analysis, by choosing specific regions where alterations were expected according to a previous report. ${ }^{4}$ The ROIs positioned in the CC, corticospinal tract, and OR had areas of $0.25,0.15$, and $0.29 \mathrm{~cm}^{2}$, respectively. Then the ROIs were automatically propagated within OsiriX software to all DKI and DTI maps.

Statistical analysis was performed with the $\mathrm{R}$ statistical computing software (http://www.r-project.org). Normality was tested by applying the Kolmogorov-Smirnov test. The Cronbach $\alpha$ was used to evaluate the interrater reliability. Group analysis (Student $t$ test) was performed to test for differences between the control and NMO groups. $P<.05$ was considered statistically significant. 


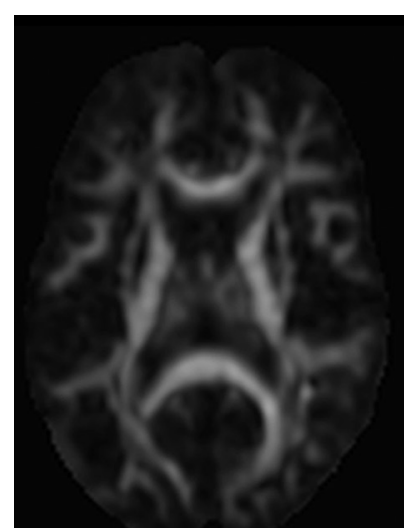

FA

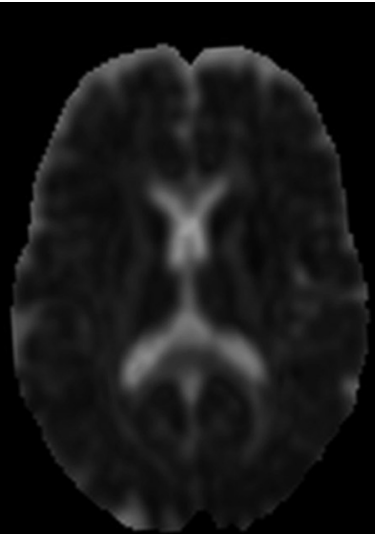

AD

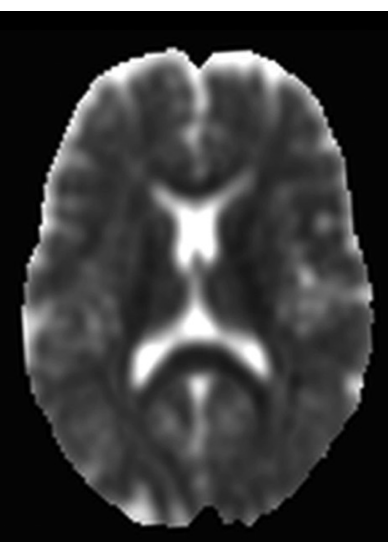

RD

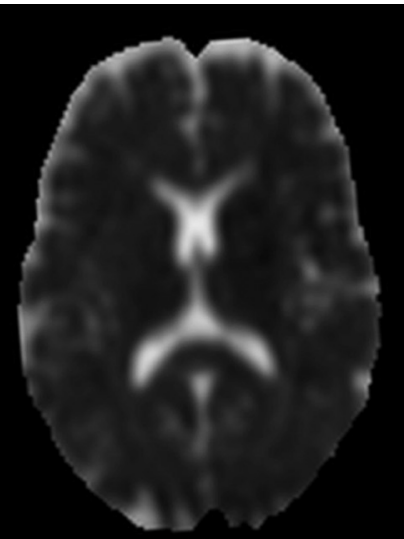

MD

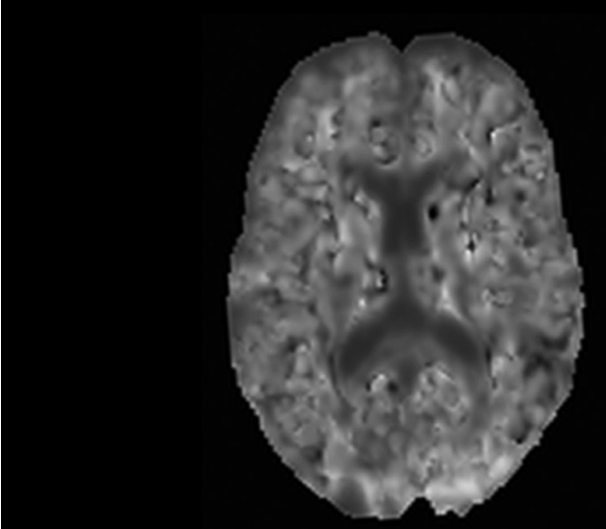

AK

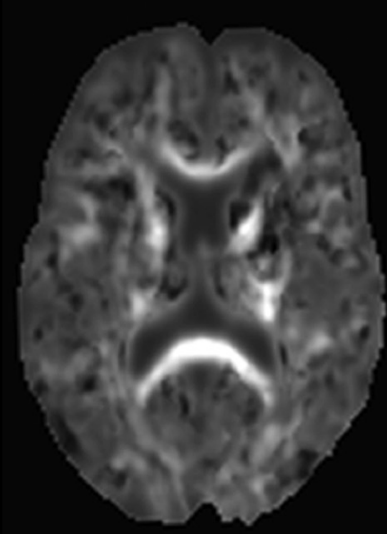

RK

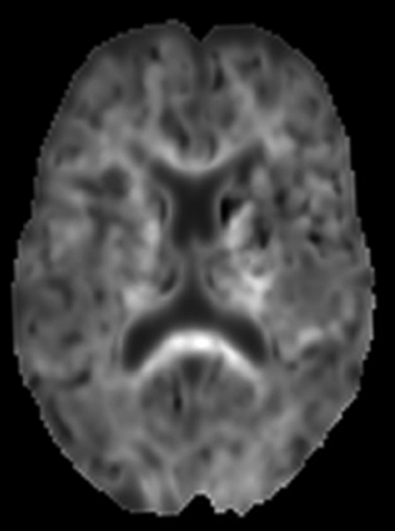

MK

FIG 1. Parametric DKI maps of 1 subject. Common diffusion parameters FA, AD, RD, and MD (upper row). Diffusional kurtosis parameters AK, RK, and MK (lower row).

Correlation between DTI and DKI Parameters. To compare conventional diffusion and DKI parameters, we calculated the Pearson correlation coefficient for all ROIs defined in the second analysis of each subject that were drawn by 1 rater (T.M.D.). A correlation coefficient of $r>0.7$ was considered a strong correlation, and a coefficient of $0.5<r<0.7$ was considered a moderate correlation.

\section{RESULTS}

\section{Tract-Based Spatial Statistics}

DTI. All results are listed in the On-line Table and illustrated in Fig 2. Reductions in FA were found in the CC (splenium and body), bilateral ORs, inferior fronto-occipital and superior longitudinal fascicules, and left internal capsule (all $P<.05$ ). MD and $\mathrm{RD}$ were increased in the CC (splenium, body, and genu); bilateral ORs; anterior, posterior, and superior corona radiata; inferior fronto-occipital and superior longitudinal fascicules; and bilateral corticospinal tracts in the frontal lobe (all $P<.05$ ). AD was increased in the CC (splenium, body, and genu), left anterior corona radiata, bilateral superior longitudinal fascicules, and bilateral ORs (all $P<.05)$.

DKI. RK was decreased in the splenium, body, and genu of the CC $(P<.05)$ and the right anterior corona radiata (Fig 2$)$. A tendency $(P<.1)$ for RK reduction was observed in both ORs. Tendencies for MK reduction $(P<.1)$ were found in the body and genu of the $\mathrm{CC}$ and in the superior corona radiata. AK showed no significant alteration.

\section{Region-of-Interest Analysis}

The results of Cronbach $\alpha$ to evaluate the interrater reliability are listed in the Table. All values showed acceptable, good, or excellent interrater reliability ( $\alpha \geq 0.7$ ), expect for 2 values. One value was questionable ( $\alpha=.6432$, right corticospinal tract, MD), and one was poor $(\alpha=.5438$, left corticospinal tract, $\mathrm{MD})$.

The On-line Table lists the results of group comparisons. Only regions in which the parameters showed a significant increase or decrease or altered tendency are shown, and only significant $P$ values with an underlying normal distribution are reported.

DTI. Rater 2 found that FA was reduced in the left OR of patients with NMO compared with controls $(P<.05)$. Both raters found that MD was increased $(P<.05)$ in the genu of the CC in patients with NMO. $\mathrm{RD}$ and $\mathrm{AD}$ were increased in the same regions $(P<.05)$.

DKI. In patients with NMO, both raters found that MK was decreased in the left OR $(P<.05)$. Rater 1 found that MK was increased $(P<.05)$ in the corticospinal tract of the right peduncle, 
whereas rater 2 observed only a tendency of increase $(P=.056)$ compared with controls. Both raters found that RK was decreased in the left OR $(P<.05)$. Rater 1 found that RK was decreased in the CC splenium $(P<.05)$, whereas rater 2 found the same result in the genu of the CC $(P<.05)$. No significant alterations in AK were observed.

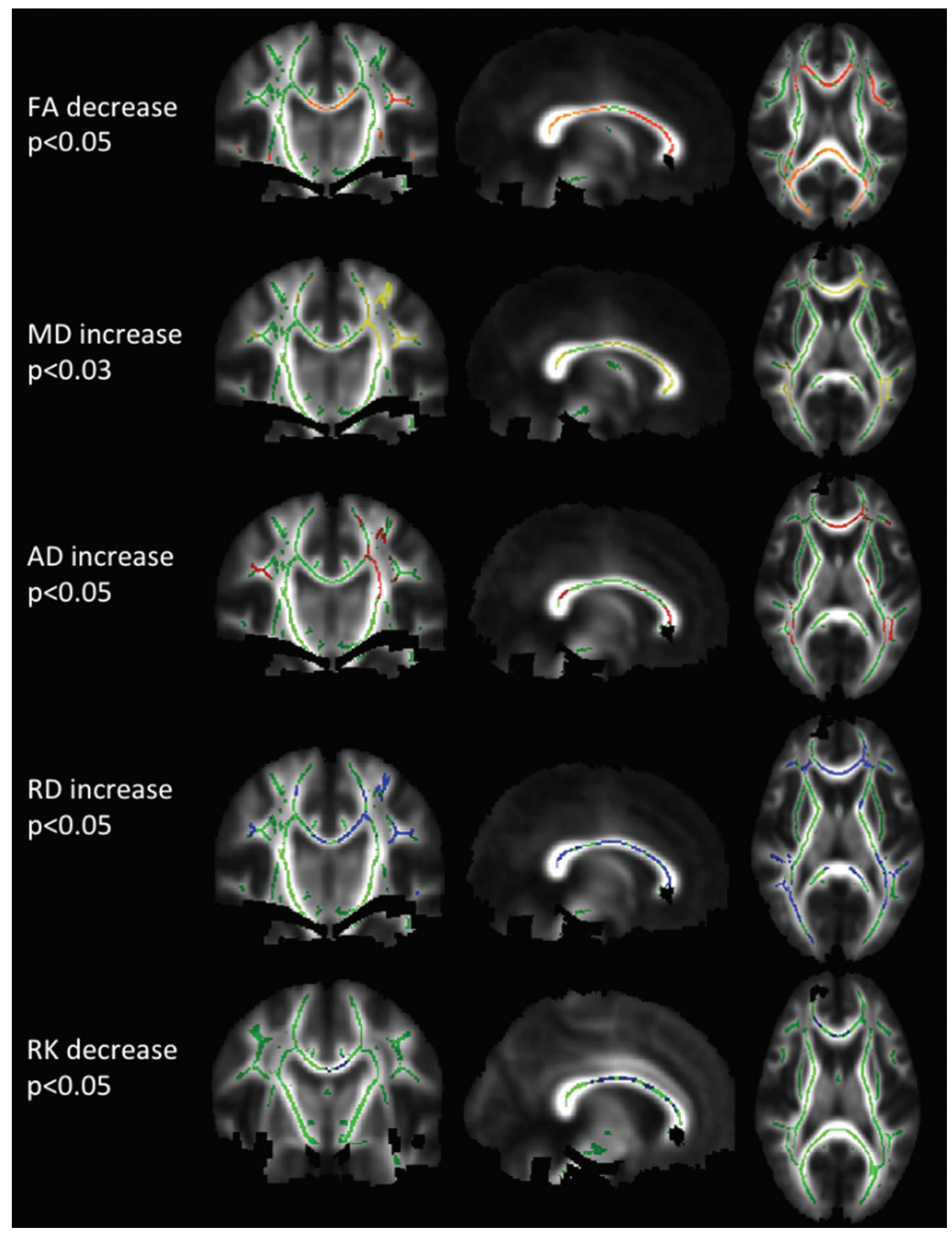

FIG 2. TBSS analysis. Significance $P$ maps, corrected for multiple comparison, for DTI and DKI parameters are overlaid color-coded on the skeleton and mean FA image. In green, the mean FA skeleton, where statistical analysis was performed. The following areas are significantly reduced in patients with NMO compared with controls: FA (red-yellow) and RK (blue), or significantly higher, MD (yellow), AD (red), RD (blue).

\section{Correlation between DTI and DKI Parameters Based on Region-of-Interest Analysis}

Strong negative correlations were found in the genu and splenium of the CC between RK and MK $(r=-0.709, P<.0001$ and $r=$ $-0.881, P<.0001$, respectively) and between MD and RD ( $r=$ $-0.752, P<.0001$ and $r=-0.821, P<.0001$, respectively). There was a moderate negative correlation between $\mathrm{AK}$ and $\mathrm{AD}$ in the bilateral ORs $(r=-0.523, P=.006$ and $r=$ $-0.584, P=.002$, respectively). A moderate positive correlation was found between FA and MK in the genu and splenium of the CC $(r=0.549, P=.003$ and $r=0.677, P<.0001$, respectively). A strong positive correlation was found between FA and RK in the splenium of the CC $(r=0.763, P<.0001)$, and a moderate positive correlation was found in the genu of the CC and the left OR $(r=0.673$, $P<.0001$ and $r=0.610 P<.001$, respectively). FA and AK were not correlated significantly.

\section{DISCUSSION}

In this study, we analyzed the WM in patients with NMO compared with healthy controls by applying the novel DKI technique, by using both TBSS (which requires no a priori hypothesis) and a region-of-interest-driven approach (which uses an a priori hypothesis). Within TBSS, several DTI and DKI parameters were altered significantly in extensive WM tracts, but the only kurtosis parameter that was altered significantly was RK. Within the region-of-interest analysis, the DTI parameters $\mathrm{FA}, \mathrm{MD}, \mathrm{RD}$, and $\mathrm{AD}$ showed significant alterations in some ROIs. The kurtosis parameters RK and MK were altered significantly. Negative correlations were found between several kurtosis and diffusion parameters. A decrease of RK corroborates the presence of demyelination. ${ }^{14}$

Lopes et $\mathrm{al}^{4}$ previously found significant changes of DTI parameters by a

Interrater reliability test for ROI-based analysis ${ }^{\text {a }}$

\begin{tabular}{|c|c|c|c|c|c|c|c|c|}
\hline & $\begin{array}{c}\text { CC } \\
\text { Genu }\end{array}$ & $\begin{array}{c}\text { CC } \\
\text { Splenium }\end{array}$ & $\begin{array}{c}\text { OR } \\
R\end{array}$ & $\begin{array}{c}\text { OR } \\
L\end{array}$ & $\begin{array}{c}\text { CST } \\
\mathbf{R}\end{array}$ & $\begin{array}{c}\text { CST } \\
L\end{array}$ & $\boldsymbol{\alpha}$ & Interpretation \\
\hline FA & 0.9604 & 0.9958 & 0.9265 & 0.9374 & 0.8504 & 0.9494 & $\alpha \geq .9$ & Excellent \\
\hline MD & 0.9110 & 0.9493 & 0.8010 & 0.7811 & 0.6432 & 0.5438 & $.8 \leq \alpha<.9$ & Good \\
\hline$A D$ & 0.9292 & 0.9299 & 0.8983 & 0.9440 & 0.9290 & 0.9829 & $.7 \leq \alpha<.8$ & Acceptable \\
\hline RD & 0.9134 & 0.9506 & 0.8401 & 0.8776 & 0.8415 & 0.7905 & $.6 \leq \alpha<.7$ & Questionable \\
\hline MK & 0.8635 & 0.9367 & 0.7894 & 0.8703 & 0.9294 & 0.8332 & $.5 \leq \alpha<.6$ & Poor \\
\hline AK & 0.9072 & 0.9526 & 0.8589 & 0.7864 & 0.8164 & 0.7967 & $\alpha<.5$ & Unacceptable \\
\hline RK & 0.8968 & 0.9407 & 0.8669 & 0.9446 & 0.9495 & 0.9611 & & \\
\hline
\end{tabular}

Note:-CST indicates corticospinal tract; R, right; L, left.

${ }^{a}$ The Cronbach $\alpha$ for each ROI, DTI, and DKI parameter is listed. 
TBSS analysis in patients with NMO. They observed significant reductions in FA in the CC, ORs, internal and external capsules, corona radiata, and cerebral peduncles and significant increases in RD in the CC, corona radiata, and centrum semiovale. They also found a highly significant negative correlation between FA and RD $(r=-0.976, P<.0001)$. In the present study, we found a significant FA reduction in those regions, except for the corticospinal tract in the cerebral peduncles. We detected a significant increase in $\mathrm{RD}$ in the $\mathrm{CC}$, corona radiata, and centrum semiovale. We found a strong negative correlation between FA and RD in all ROIs, except in the left OR, in which the correlation was moderate and negative. We found a strong positive correlation between FA and RK in the splenium of the CC and moderate positive correlations in the genu of the $\mathrm{CC}$ and the left OR. These findings support the hypothesis that the WM damage in patients with NMO is more related to demyelination (RK) than axonal degeneration (AK). This hypothesis was previously stressed in histologic studies, ${ }^{19,20}$ which showed that the demyelination process could take place even before axonal death. ${ }^{20}$

Cheung et $\mathrm{al}^{14}$ demonstrated that RK is highly sensitive to brain maturation due to myelination and, therefore, to its inverse effect, demyelination. Myelin sheath reduction increases the possibility of water diffusion in a direction perpendicular to the nerve fibers, thereby increasing RD and decreasing RK. We observed a significant decrease in RK in the CC and ORs ( $P=.028$ and $P=.011$ for raters 1 and 2, respectively, by region-of-interest analysis; $P<.1$ by TBSS). The observed strong negative correlation between $\mathrm{RK}$ and $\mathrm{RD}$ in the genu and splenium of the CC supports the hypothesis that demyelination is related to a decreased RK and increased RD. Furthermore, MK was increased in the corticospinal tract at the peduncle $(P=.047$ and $P=.56$ by raters 1 and 2 , respectively; there was no TBSS result in this area) (without representation on MD maps). This finding might be related to an initial inflammatory process in which high cellularity could restrict overall diffusion, with the diffusion distribution becoming more non-Gaussian and the MK increasing.

Recently, Lätt et $\mathrm{al}^{15}$ provided estimates of DKI for various cerebral regions in healthy brains and compared them with DTI parameters. They found a strong positive correlation between MK and FA in WM $\left(r=0.81, P<10^{-5}\right)$ and a strong negative correlation between $\mathrm{RK}$ and $\mathrm{RD}\left(r=-0.95, P<10^{-5}\right)$. In the present study, we found a moderate positive correlation between MK and FA and a strong negative correlation between RK and RD in the splenium and genu of the CC.

Our study had some limitations. During the skull-stripping process by the DKI software, regions in the cerebral peduncle were misclassified as skull in some subjects. In TBSS, after averaging the subject's images to generate a mean FA image, black regions appeared in some cases, and cross-subject statistical analysis was compromised. Unfortunately, the sensibility threshold that determines the degree of skull-stripping was not adjustable within the software version used to reconstruct the DT and DK parametric maps. This aspect of the TBSS analysis might explain why TBSS was unable to detect the significant increase of $\mathrm{MK}$ in the right corticospinal tract in the cerebral peduncle.
Another limitation may be the incorrect placements of ROIs during region-of-interest-driven analysis. However, we included this analysis because it is based on a priori data and allowed the comparison with results of previous studies because a TBSS analysis of DKI had not been performed to date, to our knowledge. Moreover, the results of the region-of-interest analysis could verify the TBSS results. The limitation of the region-of-interest analysis (lack of multiple-comparison correction) was overcome by the TBSS analysis by doing a family-wise error multiple comparison correction.

Another limitation was the suboptimal acquisition resulting from the sequence applied, which consisted of b-values of only 1 $b=0,30 b=1000$, and $30 b=2000$. Noise is a general problem in diffusion MR imaging acquisitions, and its effects have been investigated extensively. ${ }^{21}$ Using higher b-values in DKI acquisitions increases the sensitivity of the measurement to noise and, therefore, the possibility that the diffusion and kurtosis parameter estimates will vary from their true values, causing erroneous inferences. A better understanding of the effects of noise on DKI would be helpful in interpreting DKI results. Finally, because $\mathrm{NMO}$ is an uncommon disease, the patient population is quite heterogeneous when considering clinical data and treatment options; this heterogeneity is another limitation to our study, and these findings should be replicated in a different group to validate our results.

\section{CONCLUSIONS}

This study demonstrated significant differences in diffusion and kurtosis parameters in patients with NMO compared with healthy controls. Conventional diffusion parameters such as FA, MD, RD, and $\mathrm{AD}$ were more sensitive to the disease than kurtosis parameters. Only RK was significantly reduced, while MK presented a reduction tendency. AK did not show alterations. Kurtosis parameters were inversely correlated with the corresponding diffusion parameters. RK and MK correlated positively with FA. Further studies are necessary to prove the benefit of DKI parameters as biomarkers for patients with NMO.

\section{ACKNOWLEDGMENTS}

The authors would like to thank Dr Ali Tabesh for his technical assistance.

\section{REFERENCES}

1. Wingerchuk DM, Lennon VA, Pittock SJ, et al. Revised diagnostic criteria for neuromyelitis optica. Neurology 2006;66:1485-89

2. Aboul-Enein F, Krssák M, Höftberger R, et al. Diffuse white matter damage is absent in neuromyelitis optica. AJNR Am J Neuroradiol 2010;31:76-79

3. Pires CE, da Silva CMC, Lopes CRF, et al. Brain MRI abnormalities in Brazilian patients with neuromyelitis optica. J Clin Neurosci 2012;19:969-74

4. Lopes CRF, Doring TM, Martins C, et al. The role of demyelination in neuromyelitis optica damage: diffusion tensor MR imaging study. Radiology 2012;263:235-42

5. Rueda-Lopes CR, da Cruz LC, Doring TM, et al. Diffusion-weighted imaging and demyelinating diseases: new aspects of an old advanced sequence. AJR Am J Roentgenol 2014;202:W34-42

6. Tabesh A, Jensen JH, Ardekani BA, et al. Estimation of tensors and tensor-derived measures in diffusional kurtosis imaging. Magn Reson Med 2011;65:823-36

AJNR Am J Neuroradiol 35:2287-92 Dec 2014 www.ajnr.org 
7. Jensen JH, Helpern JA, Ramani A, et al. Diffusional kurtosis imaging: the quantification of non-Gaussian water diffusion by means of magnetic resonance imaging. Magn Reson Med 2005; 53:1432-40

8. Lu H, Jensen JH, Ramani A, et al. Three-dimensional characterization of non-Gaussian water diffusion in humans using diffusion kurtosis imaging. NMR Biomed 2006;19:236-47

9. Jensen JH, Helpern JA. MRI quantification of non-Gaussian water diffusion by kurtosis analysis. NMR Biomed 2010;23:698-710

10. Lazar M, Jensen JH, Xuan L, et al. Estimation of the orientation distribution function from diffusional kurtosis imaging. Magn Reson Med 2008;60:774-81

11. Tuch DS, Reese TG, Wiegell MR, et al. Diffusion MRI of complex neural architecture. Neuron 2003;40:885-995

12. Tuch DS. Q-ball imaging. Magn Reson Med 2004;52:1358-72

13. Hui ES, Cheung MM, Qi L, et al. Towards better MR characterization of neural tissues using directional diffusion kurtosis analysis. Neuroimage 2008;42:122-34

14. Cheung MM, Hui ES, Chan KC, et al. Does diffusion kurtosis imaging lead to better neural tissue characterization? A rodent brain maturation study. Neuroimage 2009;45:386-92
15. Lätt J, Nilsson M, Wirestam R, et al. Regional values of diffusional kurtosis estimates in the healthy brain. J Magn Reson Imaging 2013;37:610-18

16. Fung SH, Roccatagliata L, Gonzalez RG, et al. MR diffusion imaging in ischemic stroke. Neuroimaging Clin N Am 2011;21:345-77, xi

17. Van Cauter S, Veraart J, Sijbers J, et al. Gliomas: diffusion kurtosis MR imaging in grading. Radiology 2012;263:492-501

18. Smith SM, Johansen-Berg H, Jenkinson M, et al. Acquisition and voxelwise analysis of multi subject diffusion data with tract-based spatial statistics. Nat Protoc 2007;2:499-503

19. Lucchinetti CF, Mandler RN, McGavern D, et al. A role for humoral mechanisms in the pathogenesis of Devic's neuromyelitis optica. Brain 2002;125(pt 7):1450-61

20. Marignier R, Nicolle A, Watrin C, et al. Oligodendrocytes are damaged by neuromyelitis optica immunoglobulin $\mathrm{G}$ via astrocyte injury. Brain 2010;133:2578-91

21. Farrell JA, Landman BA, Jones CK, et al. Effects of signal-to noise ratio on the accuracy and reproducibility of diffusion tensor imaging-derived fractional anisotropy, mean diffusivity, and principal eigenvector measurements at 1.5T. J Magn Reson Imaging 2007;26:756-67 